\title{
José MARÍA ARGUEDAS Y JOÃo GUIMARÃES ROSA FRENTE A LOS ABISMOS DE LA TRADUCCIÓN
}

\author{
JoSÉ MARÍA ARGUEDAS AND JOÃo GUIMARÃES ROSA FACE TO THE \\ ABYSSES OF TRANSLATION
}

\section{JoSÉ MARÍA ARGUEDAS E JOÃO GUIMARÃES ROSA FACE AOS ABISMOS DA TRADUÇÃO}

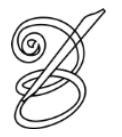 \\ Romulo Monte ALTO* \\ Universidade Federal de Minas Gerais, Brasil
}

\begin{abstract}
Resumen: A partir de los escritos y correspondencias de dos escritores considerados transculturadores, José María Arguedas y João Guimarães Rosa (RAMA, 1989), este texto busca reflexionar sobre los abismos que se plantean desde la traducción de obras literarias en el portugués brasileño y el castellano, en su variante andina. Desde los apuntes de Julio Prieto (2008) y Sanches Baena (2008) la pregunta sobre el estilo en los dos escritores aparece recortada por un agudo sentido de la oralidad con que redactaron sus textos, y que la traducción intentó recuperar en las lenguas de llegada. El examen detenido de las estrategias usadas por ellos para pensar la traducción además de desvelar otras dimensiones de su creación pone en jaque algunos conceptos esenciales al trabajo traductor, como la relación original y copia y, la fidelidad y originalidad de los textos de partida.
\end{abstract}

Palabras clave: Traducción literaria. Andes peruanos. Sertón brasileño. Abismos.

Abstract: Based on the writings and correspondence of two writers considered as promoters of transculturation of Latin American literature, José Maria Arguedas and João Guimarães Rosa (RAMA, 1989), this text seeks to reflect on the abysses that appear in the translation of literary works between Brazilian Portuguese and Spanish in its Andean variant. Based on Julio Prieto's (2008) and Sanches Baena's (2008) notes, the question about the style of the two writers is permeated by the acute sense of orality used in their texts and tried to be recovered in the translations. The careful examination of the strategies used by them to think of the translation, in addition to revealing other dimensions of their creation, calls into question certain concepts that are essential to the translator's work, such as the relation between original and copy, the fidelity and originality of the starting texts. Key words: Literary translation. Peruvian Andes. Brasilian sertão. Abysses.

Resumo: A partir dos textos e correspondências de dois escritores considerados transculturadores da literatura latino-americana, José María Arguedas e João Guimarães Rosa (RAMA, 1989), este texto busca refletir sobre os abismos que aparecem na tradução de obras literárias no português brasileiro e no castelhano, em sua variante andina. Partindo das notas de Julio Prieto (2008) e Sanchez Baena (2008), a pergunta sobre o estilo na obra dos dois escritores aparece entrecortada por um agudo sentido da oralidade com a qual eles escreveram seus textos, oralidade essa que a tradução procurou recuperar nas línguas de chegada. O exame minucioso das estratégias usadas por eles para pensar na tradução revela, além de outras dimensões dos processos criativos de ambos, alguns conceitos essenciais ao trabalho tradutor, como a relação original e cópia, bem como a fidelidade e originalidade dos textos de partida.

Palavras-chave: Tradução literária. Andes peruanos. Sertão brasileiro. Abismos.

RECEBIDO EM: 06/05/2019

ACEITO EM: 25/08/2019

PUBLICADO EM: 10/2019

ALTO, Romulo Monte. José María Arguedas y João Guimarães Rosa frente a los abismos de la traducción. Belas Infiéis, Brasília, v. 8, n. 4, p. 55-67, 2019. 
uisiera empezar este texto evocando la imagen de la carretera que se ha construido entre Brasil y el Perú, llamada Transoceánica en la parte brasileña e Interoceánica Sur y Norte en el lado peruano ${ }^{1}$. Esta obra de 2,6 mil kilómetros que recorta partes de la Floresta Amazónica y la Cordillera de los Andes, de coste en torno al US\$1,8 mil millones de dólares, se construyó con recursos de los gobiernos brasileño y peruano, además del capital privado, en especial brasileño, interesado en una salida terrestre hacia el Pacífico para sus productos, lo que significa una reducción en torno a los 6 mil kilómetros de la ruta comercial hacia los mercados asiáticos (véase A rodovia Transoceânica, en http://www.advivo.com.br/blog/luisnassif/a-rodovia-transoceanica). La figura de la carretera, sus sinuosas ondulaciones y recodos en especial en el lado peruano, ofrece una imagen preciosa de la traducción como una labor que busca sortear las diferencias y distancias culturales entre pueblos, acercando y despertando mundos antes alejados entre sí. Voy, por lo tanto, a hacer uso de una anécdota que se dio en la misma carretera, un partido de fútbol entre muchachos peruanos y brasileños en la ciudad de Assis Brasil el día 9 de noviembre de 2010, en un campo que se ubicaba junto al puente que llaman Ponte da Integração. Las imágenes de dicho partido 56 que organizó la Fundación Odebrecht, brazo peruano de la constructora brasileña encargada de la construcción de gran parte de la obra incluso en los dos países, se pueden ver en http://www.youtube.com/watch?v=EJ9VCh7ZhK0\&list=PL4B66BB26EE67E663.

Assis Brasil es una ciudad que se ubica en el estado de Acre, antiguo territorio boliviano ingeniosamente incorporado por Brasil a lo largo de su historia expansionista, más exactamente en 1903, por artes y gracia de su cuerpo diplomático liderado por el Barón del Rio Branco; también se conoce como la Ciudad de las Tres Fronteras, pues toca con Iñapari en el Perú y San Pedro de Bolpebra en Bolivia. Además de los vecinos hispánicos, el municipio alberga en casi un cuarto de sus tierras a varias comunidades nativas, originarias de dos grandes troncos lingüísticos, los Arauak, de raíz amazónica, y los Pano, de origen andina, venidos a esas tierras expulsados por los españoles; dentro de esos grupos se encuentran los Kaxinawá, Yawanawá, Katukina, Jaminawa, Kulina, Ashaninka, entre otros; además se encuentran en sus tierras las reservas destinadas a la recolección del caucho, como la Reserva Extrativista Chico Mendes, del memorable ambientalista brasileño asesinado en 1988 .

Pues bien, lo que me interesa de ese partido es un dato algo insólito que se lee en las crónicas locales, y se trata de que aparte la fiesta de integración que representó para brasileños y peruanos que viven cerca de la frontera, se utilizó en el partido la cantidad increíble de 35 balones. Eso porque se armó la cancha de fútbol cerca al puente, y claro, del río, que era el local 
donde iban a parar todos los balonazos perdidos de defensas y delanteros en sus faenas futboleras; y aunque habían 4 "gandulas", nombre dado a los chavalines que recuperan los balones en Brasil, y una piragua destinados a eso, tardaban demasiado en reponer el balón de juego, así que, a cada balón perdido, otro se tiraba a la cancha. Pues será esa imagen del abismo a que caen los balones que quería tomar para pensar la traducción entre Brasil y la América Hispánica, más precisamente entre Brasil y el Perú.

Se trata de los abismos que salvar para llevar a cabo la labor de la traducción entre las dos regiones del continente, que por supuesto no ha empezado ahora, sino más bien desde que los primeros nativos de origen Pano dejaron las tierras andinas y se fueron a vivir al Amazonas, o que el escritor romántico cuzqueño José Manuel Váldez y Palacios se volvió el primer viajero peruano a describir el país vecino, tras un largo y accidentado viaje por la selva amazónica en 1841, desde su Cusco natal hasta Belém do Pará, en Brasil, segundo Estuardo Núñez (1981). Pero por razones variadas quisiera ceñirme a la traducción entre las lenguas coloniales, el portugués y el español, y los cambios que han sufrido en tierras americanas. Son al menos tres los abismos que las separan: la lengua, el sistema literario y el tiempo histórico. Por razones de tiempo, voy a tratar solamente del primero desde algunos apuntes sobre la traducción en dos escritores que han remarcado la literatura de sendos países.

El primer abismo a que me refiero es la lengua. No la lengua común, general, estándar como dirían algunos, sino la lengua literaria que es donde se presenta con más intensidad la "carga de electricidad histórica" que conllevan las palabras y expresiones en la traducción literaria, según Julio Prieto (2008). En sus mismas palabras: "en la medida en que la temporalidad de las palabras es lingüísticamente específica, esa carga de tiempo acumulado es uno de los abismos más difíciles de salvar para la traducción" (PRIETO, 2008, p. 156). Quiero referirme no a la lengua requisitoria del Inca Garcilaso de la Vega o del Padre Vieira, ni a la barroca de Juan de Espinoza Medrano, el Lunarejo, o Gregorio de Matos, ni a la ilustrada de Ricardo Palma o Euclides da Cunha, ni a la modernista de César Vallejo o Carlos Drummond de Andrade, sino con más contemporaneidad, a la lengua de José María Arguedas y João Guimarães Rosa, dos fecundos transculturadores según Ángel Rama (1989). Hombres que, desde lugares interiores de naciones periféricas, lugares esos marcados históricamente por el atraso, como lo son los andes peruanos y el sertón brasileño, armaron un lenguaje que partía de la savia de culturas dichas arcaicas y olvidadas sobre las cuales se injertó lo universal, lo que les permitió florecer con perfume propio y revelar al mundo su sentido moderno (RAMA, 1989). Sí, creo que desde una matriz productiva de lo arcaico se puede pensar esa lengua 
literaria que dichos autores van a consagrar, en especial en las obras Los ríos profundos (1958) y Grande sertão: veredas (1956), como la lengua fundacional de un momento de la historia de la literatura y la cultura de su país. Veamos como esos dos escritores traductores se enfrentan a esa "carga de tiempo acumulado", según Prieto, cuando lidian con sus propios textos u otros en la traducción y si podemos con ello trenzar algunos puentes sobre los abismos mencionados.

En 27 de noviembre de 1962, Alexandra von Miquel, de la editorial Verlag Kiepenheuer \& Witsch, en Colonia, Alemania, escribe una carta a Arguedas en que le envía una copia del contrato de la traducción de Los ríos profundos para su conocimiento y firma. En dicha carta, al contarle de su impresión tras leer el ejemplar que el escritor le enviara con las correcciones, von Miquel sin saberlo toca en el tema de los límites entre las lenguas. Dice así en la carta:

permítame decirle que he estado extraordinariamente impresionada por la lectura de esta novela tan bien escrita y en la cual se revela, como me parece, una gran fuerza épica. Para el lector, una gran fascinación emana del personaje Ernesto, de sus aventuras, del medio, de todo ese mundo tan complejo en el cual se desenvuelve el muchacho. Como no sé (sic) suficientemente la lengua española, no puedo sino hacerme una vaga idea del estilo de su novela. Pero siento muy bien que su estilo, que su lenguaje, es de gran calidad y de una rara belleza. Espero con mucha impaciencia la traducción alemana. Hay que encontrar el mejor traductor para este libro. Esto no es fácil, lo sé. Sería muy bueno que el traductor conociese un poco el medio de la novela, pero sería esencial que lograse transportar su estilo, el timbre del original, a la lengua alemana. Usted me permitirá dar su dirección al traductor para que pueda dirigirse a usted en caso de dificultades.

$[\ldots]$

Claro que aquí hay excelentes traductores para la lengua española. En todo caso, le aseguro que haré todo lo posible por destinar a su novela el mejor traductor. Si aprueba el contrato, le ruego retornarnos un ejemplar firmado por usted y guardar usted el otro. (PINILLA, 2007, p. 315-316; los subrayados son nuestros).

La señora Alexandra sabe de sus limitaciones para entender la obra que tiene en manos, por eso apela a expresiones más cercanas a la sensibilidad de una editora para hacer frente al conocimiento que se le escapa: "me parece", "no puedo sino hacerme una vaga idea", "siento muy bien", son términos a los cuales recurre con la finalidad de expresar su opinión sobre la novela. Espera por la traducción al alemán para que al fin pueda comprobar sus impresiones y reitera el compromiso de buscar "el mejor traductor" para la obra en dos momentos de la carta, una insistencia que no pasa inadvertida. La razón para hacerlo dos veces no se la explica, aunque podemos imaginarla: ella no busca un traductor sino el mejor, el que sepa "transportar su estilo". La palabra estilo se repite en tres ocasiones en la carta, como algo que seguramente cobrará bastante relevancia para la autora de la misiva. Al parecer es el meollo del criterio que von Miquel usa para definir una buena labor traductora, que entienda cómo hacer escuchar "el 
timbre del original" en la lengua alemana. Por eso, creo que no sería demasiado arriesgado decir que "el mejor traductor" se asemeja en este caso a un buen músico. El que hará que se escuche en la lengua a la que se traduce el timbre de la lengua desde la cual se va a traducir.

Lástima que no tengamos a la autora de la carta a explicarnos qué significados le hubiera querido dar a su concepto. Pero creo que la sonoridad de una lengua frente a otra no se le habrá escapado, pues de eso se trata a la hora de intentar transportar un estilo: hacer escuchar otra música. De eso hablaba Ángel Crespo en 1967, en la nota introductoria a la traducción de el Gran sertón, en que afirmaba que su traducción buscaba "una adecuación al tono oral, y no meramente 'literal', de la obra de Guimarães Rosa" (ROSA, 1967, p. 9-11). Las palabras de Crespo me llevaron a otra correspondencia, una carta que le envió João Guimarães Rosa a su traductor italiano Edoardo Bizarri, en 1963, justo un año después de la carta de Alexandra von Miquel a Arguedas, en que lo felicita por la traducción exitosa de su cuento "Duelo" al italiano y plantea un supuesto vínculo entre los dos: "siento una correspondencia íntima, un tono anímico de familia, un parentesco entre nosotros: yo "continúo" en tu texto italiano, y, no lo dude, y en muchos pasajes siento que me superas, me sobrepasas. El ritmo, la dinámica, los timbres"2. Aunque el escritor brasileño ponga énfasis en la relación que se establece entre ellos momentos antes de que Bizarri dé inicio a la traducción de su obra Corpo de Baile (1956), como cierto modo de alentarlo, la lectura atenta de la correspondencia entre ellos revela la importancia de la sonoridad en la elección y creación de los vocablos por Guimarães Rosa, pero más aún en la traducción de Bizarri al italiano. En otra carta meses después, al contestar una pregunta sobre la traducción de los nombres, el escritor afirma: “exacto. Así también lo pensaba yo: tú dejando a unos como están, y traduciendo a otros. O, igual, 'inventando'. Cuando entra tu 'criterio exclusivamente personal, arbitrario y fónico', me siento alegre y tranquilo. En él es que yo, sinceramente, confío"3. Entre los criterios para la invención o creación traductora el elemento fónico se destaca: para que el escritor continuara en el texto en italiano, era esencial que la creación traductora pudiera recuperar la sonoridad del texto. Esa misma sonoridad se repetirá en las explicaciones futuras del escritor sobre su modo de creación literaria.

Un viaje a Chimbote en enero de 2011, para tomar parte en un homenaje que se hizo a Arguedas por el centenario de su nacimiento, me brindó la oportunidad de conocer la ciudad que describía, un poco como decía el escritor al escribir su última novela: "creo no conocer bien a las ciudades y estoy escribiendo sobre una". La estancia en esta ciudad me permitió escuchar los ruidos del puerto que hoy se resiente de la caída del movimiento pesquero, el trajín callejero de una ciudad empobrecida que al parecer desconoce la diferencia entre días laborales y fines 
de semana, el sordo ir y venir del viento que empuja de un lado a otro la fina arena que te toca el pantalón del viajero, el silencio que recorta el alto horno de la fábrica de aceros (hoy propiedad de del grupo brasileño Gerdau) ya casi sin vida: todo eso me tomó parte del tiempo en la aquella ciudad, adonde fui con ganas de escuchar los ruidos, los timbres que emanan de sus calles y avenidas y bares, donde todavía las personas mantienen la esperanza de días mejores al recordar los lejanos y alucinantes años del boom de la pesca (en un texto publicado en la revista Bellamar, de la Universidad del Santa, Chimbote, n. 21, en diciembre 2011, describí con más afectividad esta estancia en Chimbote).

Escuchar. Quizás traía al oído las palabras de José Martí: “es bueno que al mismo tiempo que traduzcas, aunque no por supuesto a la misma hora, leas un libro escrito en castellano útil y sencillo, para que tengas en el oído y en el pensamiento la lengua en que escribes" (MARTÍ, 1975). Escuchar en mi caso suponía reconocer en la literatura brasileña a una voz y una lengua familiar a lo que traducía. Otra vez volvía la voz de Guimarães Rosa, ahora señalando la ruta a Bizarri: “tú no eres solo un traductor. Somos 'socios', eso sí, y la invención y creación deben ser constantes"4. Bizarri por su turno le contesta: “intenté darle el ritmo, la rima, el gusto de los acercamientos inesperados, el sentido general y gracioso del absurdo anhelo humano, escapando necesariamente de una traducción al pie de la letra"5. Guimarães le señala su método de composición: "pero, el verbo sarajava, lo escuché, y el contador no supo explicarme qué significa. Verbo solo en aa, bellísimo !"6, revelando que no sabía el significado de varios de los términos y expresiones que utilizara. Le interesaba su sonido más que su sentido. El traductor padece su desconsuelo: "dudo que las relaciones de nombres de plantas y bichos, así como los gritos de los vaqueros, puedan tener traducción posible en otra lengua. [...] ¿Qué hacer?”7. Luego el maestro de la literatura brasileña le explica de forma didáctica: "para no quedar deltodo arbitrarias las representaciones imaginadas o "entrevistas” en aquel revolotear fantomático de polvo espectral - y una vez que se trata de observador tosco, rocero inculto, - algunos elementos básicos, estos, sí, se han utilizados: a moda o ad instar de "caballo-de-injerto", de planta rústica que sirve para recibir los injertos exóticos" $"$. Estamos frente a nada menos que a la explicación sobre la manufactura del proceso de la transculturación antevisto por Rama, que supone que, al tronco de los elementos rústicos, naturales de la cultura, se injerta lo que viene de afuera. Guimarães le brinda al traductor unas pautas traductoras muy claras: "tú podrás disfrutar de más libertad. Para recalcar más, lo que encuentre necesario. Para omitir lo que, en una traducción, venga a mostrarse inútil experiencia. Para dejar a un lado lo que sea intraducible, o resumir, depurar, concentrar"9. Termina el escritor brasileño su arenga 
traductora con una confesión, quizás para seguir alentando al traductor que se encontraba sin ánimo frente a los desafíos encontrados:

\begin{abstract}
pero, para que mejor te tranquilices, te digo la verdad. Yo, cuando escribo un libro, lo voy haciendo como si lo estuviera "traduciendo", desde algún alto original, existente en otra parte, en el mundo astral o en el "plano de las ideas", de los arquetipos, por ejemplo. Nunca estoy seguro de acertar o fallar, en esa "traducción". Así, cuando me "re-traducen a otro idioma, nunca estoy seguro, también, en casos de discordancia, si no fue el Traductor quien, de hecho, acertó, y restableció la verdad del "original ideal", que yo desvirtuara (ROSA, 2003, p. 99) ${ }^{10}$.
\end{abstract}

El escritor asume la insegura posición del escritor como un traductor que nunca está seguro de que su texto haya traducido a plenitud lo que haya visto o sentido desde un plan original; en verdad, sus palabras ponen en jaque la relación entre original y copia, al revelar el débil vínculo entre el significado y el significante de los difíciles vocablos que elegía para sus textos, que no se asentaban en una correspondencia que no fuera de orden musical o incluso accidental. El golpe final viene con una insólita autorización que le extiende al traductor Bizarri, para que interfiera en su texto con el fin de usar toda su libertad. Al final remata con las palabras: "lo importante es enriquecer la cosa con "humor", menos importante la estricta equivalencia"11. En estas palabras se puede desvelar todo un programa de traducción: la creación o invención son elementos rectores en dicho programa, articulados en torno a indicadores como "acentuar", “omitir", “dejar", "resumir", “enriquecer".

Volvamos a Arguedas.

Arguedas es un traductor singular. Son dos los principales escritos donde registra lo que piensa de la traducción: el primero, Un ensayo sobre la capacidad de creación artística del pueblo mestizo e indio, en Canto Kechwa, un libro publicado en 1938 en que recoge y traduce al castellano una serie de músicas andinas; y segundo, Introducción a la primera edición del manuscrito Dioses y hombres de Huarochirí, del Padre Francisco de Ávila, editado en 1966. En el primer texto el escritor traductor hace hincapié de que su traducción no es literal y que ha buscado lo poético de los versos, además de entregar sus pautas de trabajo:

no he hecho traducciones literales, he hecho versiones poéticas, el tema de las canciones está puro y entero. En "Sin día, sin nadie..." me he tomado la libertad de crear una metáfora - subrayada - que no está expresa en el verso kechwa, con el objeto de igualar la fuerza poética del último cuarteto de esa canción. En "Dile que he llorado..." he aumentado el primero y el último pie, para describir al picaflor siwar que es el tema de la canción. Publico dos traducciones de la canción del incendio porque creo que apenas, ambas juntas, dan una versión de la fuerza expresiva del canto en kechwa; la segunda es más fiel. Por último, el segundo pie de "Raki-ra-ki" es una 
interpretación del tema y del símbolo, porque esos versos son casi intraducibles. (ARGUEDAS, 1938, p. 17).

Arguedas relaciona sus operaciones: hace versiones poéticas, crea metáforas, aumenta versos, hace dos versiones de una misma canción (y según una alumna que ha hecho la traducción al portugués del Canto Kechwa como su trabajo final de conclusión de carrera, hubo una tercera versión $)^{12} \mathrm{y}$, por fin, interpreta libremente lo que le parece intraducible. Termina reiterando que las traducciones no son "rigurosamente literales, son traducciones un tanto interpretativas, que quizá desagradarán un poco a los filólogos, pero que serán una satisfacción para los que sentimos el kechwa como si fuera nuestro idioma nativo" (ARGUEDAS, 1938, p. 18). Opone al pensamiento racional, de los "filólogos", el sentimiento, la "satisfacción", de enfrentarse a un texto en que una persona pudiera reconocerse. Esa perspectiva va a madurar con el paso de los años y sobretodo con los estudios etnográficos, pero nunca cambiar.

En la "Introducción” al Manuscrito de Huarochirí, recuerda que realiza la traducción de una “traducción”, ya que Francisco de Ávila también traducía los relatos de los informantes, lo que de algún modo responde por los equívocos tanto suyos como los de Ávila. Insiste en el carácter oral del texto: "el estilo del manuscrito es predominantemente oral" (ARGUEDAS, 2009, p. 2), a fin de justificar su misma traducción, que será necesariamente distinta al “castellano limpio y florido" de Ávila, lo que no le permitió que "escribiera el texto tan oral y espontáneo del manuscrito" (ARGUEDAS, 2009, p. 5), pues espera que el lector pueda sentir el "torrente del lenguaje" de la obra.

La música una vez más toma el centro del texto arguediano. Desde las historias de la hacienda Viseca, en que los peones, mujeres y niños cantaban juntos a los señores, hasta el incomprensible silencio que reinaba en la hacienda de su tío en el valle del Apurímac, el autor anuncia: "el wayno es arte, como música y como poesía", seguido de la reivindicatoria: "solo falta que se haga ver bien esto" (ARGUEDAS, 2009, p. 13). Si la crítica ha demostrado que la oralidad está en el corazón de la escritura ficcional arguediana (CORNEJO POLAR, 1997; LIENHARD, 1990; ROWE, 1996), no sería demasiado arriesgado decir que también ordena su trabajo traductor, como se puede acotar en el Estudio Preliminar que Luis Millones escribe para la reedición de Dioses y hombres de Huarochirí, en 2009. Millones rebate la idea de que la traducción de Arguedas del manuscrito fuera "más poética o menos literal que otra" (MILLONES, 2009, p. 18), sino que recibe un nuevo tratamiento del escritor y etnógrafo, que la contextualiza entre los rituales religiosos conocidos, lo que permite con eso recobrar la perdida oralidad del texto original. De algún modo, Arguedas actúa en la traducción como 
pronostican los estudiosos de la llamada Escuela de la Manipulación (SANCHES BAENA, 2008), que señalan el papel activo y manipulador del traductor según supuestos ideológicos; el escritor traductor resalta repetidas veces la necesidad de que el arte (y dentro de ella la traducción) ofreciera una obra que no simplemente fuera entendida, sino más bien, que fuera “capaz de conmover al hombre a quien la obra se refiere" (ARGUEDAS, 1938, p. 14), o el futuro lector en quechua que espera que lea su obra.

El artículo La novela y el problema de la expresión literaria en el Perú se publica por primera vez en la revista Mar del Sur, en 1950, y se reproducirá posteriormente, encabezando las reediciones de 1968 de Yawar Fiesta, por la Editorial Universitaria de Santiago de Chile, y también en las Obras Completas que publica la Editorial Horizonte en 1985, además de aparecer en la recopilación de textos que organiza Juan Larco para Casa de las Américas (La Habana, 1976). En este texto, Arguedas se plantea el tema del estilo como algo al parecer insoluble: “¿cómo describir esas aldeas, pueblos y campos; en qué idioma narrar su apacible y a la vez inquietante vida? ¿En castellano? ¿Después de haberlo aprendido, amado y vivido a través del dulce y palpitante quechua?” (ARGUEDAS, 1985, p. 195). A continuación, cuenta del sentimiento de fracaso que sintió en su primer intento de escribir de forma literaria un cuento y entra al debate en torno al binomio regionalismo versus universalidad. Luego afirma que su búsqueda por el estilo fue "larga y angustiosa", entendido el estilo en especial por la lengua literaria que usaría en la narración de los hechos y que tendría por finalidad transmitir "a los demás ese mundo" [serrano], así como "las extremas pasiones de los seres humanos que lo habitaban". Sus dudas giraban en torno al castellano en que escribía, pues sabía que no encontraría lectores para sus textos en quechua, una lengua predominantemente oral.

Si en el ensayo que abría Canto kechwa el énfasis recaía sobre el quechua como lengua más que suficiente para la expresión estética del hombre andino, reafirmado en la experiencia del mismo autor, en el artículo que sigue a la Nota preliminar de Yawar Fiesta (1968), Arguedas apuesta por desvelar la lucha que libró en la búsqueda de la lengua literaria con que describir aquellas realidades serranas, dejando claro su apuesta lingüística: "he fundamentado en un ensayo mi voto a favor del castellano" (ARGUEDAS, 1985, p. 197). Toda La lucha por el estilo, subtítulo del segundo apartado del artículo (seguido de las palabras Lo regional y lo universal), se resume al repaso del exhaustivo proceso de escribir y reescribir los cuentos de Agua, desde los cuales se enfrenta a la pelea con el castellano ${ }^{13}$. Tras redactar los primeros cuentos, se entera de no ser esa la lengua que necesitaba para revelar en las ciudades de abajo los mundos de arriba: “comprendí definitivamente que el castellano que sabía no me serviría si 
seguía empleándolo en la forma tradicionalmente literaria” (ARGUEDAS, 1985, p. 196). La única posibilidad que quedaba era tomar esta lengua y cambiarla: "era necesario encontrar los sutiles desordenamientos que harían del castellano el molde justo, el instrumento adecuado" (ARGUEDAS, 1985, p. 196). El tiempo que tardó su lucha: “cinco años luché por desgarrar los quechuismos y convertir al castellano literario en el instrumento único"; el precio que pagó: “yo sé que algo se pierde a cambio de lo que se gana" (ARGUEDAS, 1985, p. 197).

Sin embargo, sobre este trabajo a realizarse Arguedas plantea una ventaja al artista, que es "la posibilidad, la necesidad de un acto de creación más absoluta" (ARGUEDAS, 1985, p. 196). Al igual que Guimarães Rosa, quien conocerá años más tarde en Venecia, Italia, en el Congreso Internacional de Escritores Latinoamericanos, y aseguraba la necesidad de que el escritor, para hacer frente a la "Babel espiritual de valores" del mundo moderno, crease su mismo léxico ya que no le restaba otra opción pues de lo contrario no tendría como llevar a cabo su misión (LORENZ, 1994, p. 53), el escritor peruano relata feliz el hallazgo estético que le llegó como los sueños, de "manera imprecisa". Su afirmación conclusiva al final del artículo no deja duda de que su lucha culminaba exitosa:

creo que en la novela Los ríos profundos este proceso ha concluido. Uno sólo podía ser el fin: el castellano como medio de expresión legítimo del mundo peruano de los Andes; noble torbellino en que espíritus diferentes, como que forjados en estrellas antípodas, luchan, se atraen, se rechazan y se mezclan, entre las más altas montañas, los ríos más hondos, entre nieves y lagos silenciosos, la helada y el fuego (ARGUEDAS, 1985, p. 197).

Pero para lograr esa lengua tan deseada, el escritor tuvo que pagar un precio alto: necesitó alejarse lingüísticamente del mundo de donde venía, en cuyo perímetro al parecer no daba con el estilo que buscaba. O como se refiere Estele Tarica (2006), haciendo eco de las palabras de William Rowe (1979), nuestro autor renuncia a la poética de la "mistura" para acercarse a la poética de la traducción, que encuentra en Los ríos profundos su máxima realización, en razón de la incapacidad del lector a quien llega su obra de entender que lo que escribe no es pura mímesis de un castellano retorcido. La expresión "cinco años luché por desgarrar los quechuismos" echa luz sobre parte de su estrategia en la labor de llegar a la lengua que buscaba. Al mirar a otro lector, menos localizado e identificado con la temática serrana, señala el rumbo de su misma narrativa, que al parecer deja atrás el indigenismo como referente principal y migra, como las poblaciones serranas que se dirigen hacia Lima, camino de un mercado más ancho de intercambios culturales ${ }^{14}$. 
Guimarães Rosa y Arguedas siguen vigentes como paradigmas de una escritura en que la oralidad enmarca un territorio de expresión donde lo arcaico, lejos de señalar hacia atrás, al atraso o al olvido, proyecta un mundo repoblado de términos que al exponerse a la luz de la modernidad tomaron nuevos rumbos, fundando una nueva comunidad de sentidos. Al trenzar sus palabras, que se revelan temas provechosos para la historia de una traducción en América Latina y nos ponen de cara a la necesidad de seguir buscando el diálogo entre esos dos mundos, tan cercanos y tan distantes a la vez, creo que tenemos por delante una buena posibilidad de evitar que los balones no vuelvan a caer en el vacío. Convocar renovadas traducciones de la obra de Guimarães Rosa al español, así como las obras de Arguedas al portugués, abrirían nuevas puertas a ampliar los límites de esa reflexión.

\section{REFERENCIAS}

ARGUEDAS, José María. Canto Kechwa. Lima: Compañía de Impresiones y Publicidad Enrique Bustamante y Ballivián, 1938.

ARGUEDAS, José María. Yawar Fiesta. In: ARGUEDAS, José María. Obras completas. Tomo II. Lima: Editorial Horizonte, 1985. p. 69-227.

ARGUEDAS, José María. Introducción a la primera edición. In: ÁVILA, Francisca de. Dioses y hombres de Huarochirí. Narración quechua recogida por Francisco de Ávila [¿1598?]. Traducido por: José María Arguedas. Lima: Universidad Antonio Ruiz de Montoya, 2009.

CORNEJO POLAR, Antonio. Los universos narrativos de José María Arguedas. Lima: Editorial Horizonte, 1997.

ESCOBAR, Alberto. Arguedas o la utopía de la lengua. Lima: IEP Ediciones, 1984.

LIENHARD, Martin. Cultura andina y forma novelesca. Zorros y danzantes en la última novela de Arguedas. Lima: Editorial Horizonte/Tarea, 1990.

MARTÍ, José. Cartas de José Martí a María Mantilla. Obras Completas. v. 20. La Habana: Ed. Ciencias Sociales, 1975. p. 216-220.

MILLONES, Luis; TOMOEDA, Hiroyasu. Estudio preliminar. In: ÁVILA, Francisca de. Dioses y hombres de Huarochirí. Narración quechua recogida por Francisco de Ávila [¿1598?]. Traducido por: José María Arguedas. Lima: Universidad Antonio Ruiz de Montoya, 2009.

NÚÑEZ, Estuardo. EI Brasil visto por viajeros peruanos. Lima: Centro de Estudios Brasileños, 1981.

PINILLA, Carmen María (Ed.). Apuntes inéditos. Celia y Alicia en la vida de José María Arguedas. Lima: Fondo Editorial de la Pontificia Universidad Católica del Perú, 2007. 
PRIETO, Julio. El margen de la traducción: orillas y cruces rioplatenses. In: BLAS, Amelia et al. El reverso del tapiz. La traducción literaria en el ámbito hispánico. VII Coloquio Internacional. Instituto Cervantes: Budapest, 2008. p. 154-168.

RAMA, Ángel. Transculturación narrativa en América Latina. Montevideo: Siglo Veintiuno Editores; Fundación Ángel Rama, 1989.

ROSA, João Guimarães. Gran sertón: veredas. Trad. Ángel Crespo. Barcelona: Seix barral, 1967.

ROSA, João Guimarães. João Guimarães Rosa: correspondência com seu tradutor italiano Edoardo Bizarri. 3. ed. Rio de Janeiro: Nova Fronteira; Editora UFMG, 2003.

ROWE, William. Ensayos arguedianos. Lima: Centro de Producción Editorial de la Universidad Nacional Mayor de San Marcos/SUR Casa de Estudios del Socialismo, 1996.

ROWE, William. El castellano modificado. In: ROWE, William. Mito e ideologia en la obra de José María Arguedas. Lima: Instituto Nacional de Cultura, 1979. p. 49-63.

TARICA, Estele. El "decir limpio" de Arguedas: la voz bilíngue. 1940-1958. In: FRANCO, Sergio F. José María Arguedas: hacia una poética migrante. Pittsburgh: ILII, 2006. p. 2338 .

SÁNCHEZ BAENA, María Valentina. La escuela de la Manipulación. In: BLAS, Amelia et al. El reverso del tapiz. La traducción literaria en el ámbito hispánico. VII Coloquio Internacional. Instituto Cervantes: Budapest, 2008. p. 100-112.

\footnotetext{
* Romulo Monte ALTO - Doutor (2005) e Mestre (1999) em Estudos Literários pela Universidade Federal de Minas Gerais. Graduado em Letras (1997) pela mesma instituição. Realizou pesquisa pós-doutoral (2012-2013) na Universidad Jaume I, Espanha. É professor associado III na Universidade Federal de Minas Gerais. Belo Horizonte, Minas Gerais, Brasil.

Currículo acadêmico: http://lattes.cnpq.br/5180190290434491

ORCID: https://orcid.org/0000-0002-5859-9867

E-mail: romulomontealto@gmail.com

1 Este texto fue presentado en el Coloquio Brasil y América: rutas del siglo XX, organizado por Julio Ortega (Brown University) en Casa de América, Madrid, de ahí el lector encontrar un tono más conversacional que asertivo en torno a las ideas que desarrolla.

2 "Eu sinto que há uma correspondência íntima, um tom anímico de família, um parentesco entre nós dois: eu 'continúo', no texto seu italiano, e, não duvide, e em muitas passagens me sinto superado, ultrapassado. $O$ ritmo, a dinâmica, os timbres" (ROSA, 2003, p. 26).

3 "Exato. Assim também é que eu pensava: V. deixando uns como estão, e traduzindo outros. Ou, mesmo, 'inventando'. Quando entra seu 'critério exclusivamente pessoal, arbitrário e fônico', fico alegre e tranqüilo. Nele é que eu, sinceramente, confio" (ROSA, 2003, p. 38).

4 "Você não é apenas um tradutor. Somos "sócios", isto sim, e a invenção e criação devem ser constantes" (ROSA, 2003, p. 51).

5 "Procurei dar o ritmo, a rima, o gosto das aproximações inesperadas, o sentido geral e jocoso do absurdo anseio humano, fugindo forçosamente de uma tradução ao pé da letra" (ROSA, 2003, p. 60).

6 "Mas, o verbo sarajava, eu o ouvi, e o contador não soube explicar-me o que é. Verbo só em aa, belíssimo!" (ROSA, 2003, p. 59).

7 “duvido que as relações de nomes de plantas e bichos, e de gritos dos vaqueiros, possam ter tradução cabível em outra língua. [...] Que fazer?” (ROSA, 2003, p. 68).
} 
8 “Para não ficarem de-todo arbitrárias as representações imaginadas ou 'entrevistas' naquele revolutear fantomático de poeira espectral - e devendo tratar-se de espectador rude, roceiro inculto, - alguns elementos básicos, estes, sim, foram utilizados : à moda ou ad instar de 'cavalo-de-enxerto', de planta rústica que serve para receber os enxertos exóticos" (ROSA, 2003, p. 84).

9 "Você poderá ter mais liberdade. Para acentuar mais, o que achar necessário. Para omitir o que, numa tradução, venha a se mostrar inútil experiência. Para deixar de lado o que for intraduzível, ou resumir, depurar, concentrar" (ROSA, 2003, p. 95).

10 "Porém, para melhor tranqüilizá-lo, digo a verdade a Você. Eu, quando escrevo um livro, vou fazendo como se o estivesse 'traduzindo', de algum alto original, existente alhures, no mundo astral ou no 'plano das idéias', dos arquétipos, por exemplo. Nunca sei se estou acertando ou falhando, nessa "tradução”. Assim, quando me 'retraduzem' para outro idioma, nunca sei, também, em casos de divergência, se não foi o Tradutor que, de fato, acertou, restabelecendo a verdade do 'original ideal', que eu desvirtuara" (ROSA, 2003, p. 99).

11 “O importante é enriquecer a coisa com 'humor', menos importante a estricta equivalência” (ROSA, 2003, p. 124).

${ }^{12}$ BATISTA, Stefania Laura Pereira. A tradução de Canto Kechwa de Arguedas ao português. 2015. Trabalho de Conclusão de Curso (Graduação em Bacharelado Tradução Espanhol) - Faculdadede Letras da Universidade Federal de Minas Gerais, Belo Horizonte, 2015.

13 El examen de dichas alteraciones entre las ediciones de Agua es el tema del capítulo 3 de la obra Arguedas o la utopia de la lengua (1984), de Alberto Escobar.

14 El fragmento que se inicia en la mitad de la página 9, con "El artículo 'La novela y el problema de la expresión literaria en el Perú'[...]", hasta el párrafo final que termina con la expresión "intercambios culturales", en la página 11, ha sido extraído de un texto de mi autoría, "Arguedas y el problema del estilo en las reediciones de Yawar Fiesta", publicado en la INTI Revista de Literatura Hispánica, v. 73-74, p. 37-49, 2011, de Brown University, EEUU, con ligeros cambios. 